\title{
Lessons of the month: HLA-B27-associated syndrome and spontaneous intracranial hypotension resulting in behavioural variant frontotemporal dementia
}

\author{
Authors: Amit KJ Mandal, ${ }^{A}$ Angad Ryatt, ${ }^{B}$ Kim O'Hare ${ }^{B}$ and Constantinos G Missouris ${ }^{C}$
}

Spontaneous intracranial hypotension is uncommon and results from a cerebrospinal fluid (CSF) leak. We describe the case of a marathon runner who presented with postural headache attributable to CSF venous fistulation originating from a lower thoracic nerve root cyst. Subsequent investigations confirmed a unifying de novo diagnosis of human leukocyte antigen B27-associated syndrome. With unmitigated CSF loss over the following 3 months, the patient became bedbound and developed rapidly progressive behavioural variant frontotemporal dementia. Behavioural changes were somewhat reversible on restoration of CSF volume after surgical intervention.

KEYWORDS: Spontaneous intracranial hypotension, CSF leak, connective tissue disease, HLA-B27, behavioural variant frontotemporal dementia

DOI: $10.7861 /$ clinmed.2020-0062

\section{Case presentation}

A 63-year-old man, and competitive marathon runner, presented with a 4 -week history of disorientation and bitemporal orthostatic headache. Over the preceding 2 years, there had been issues with balance, short-term memory loss and daytime hypersomnolence.

Clinical inspection revealed tall stature with normal posture. Musculoskeletal and neurological examinations were normal. Cardiovascular examination revealed grade 2/4 aortic regurgitation.

Laboratory investigations were notable for elevated anti-double stranded deoxyribonucleic acid titre at $75.0 \mathrm{IU} / \mathrm{mL}$ and human leukocyte antigen B27 (HLA-B27) positivity.

Magnetic resonance imaging (MRI) demonstrated smooth pachymeningeal thickening (Fig 1a) with prominent perivascular

Authors: ${ }^{\text {A }}$ consultant, Wexham Park Hospital, Slough, UK; ${ }^{B}$ senior house officer, Wexham Park Hospital, Slough, UK; ${ }^{C}$ consultant and professor, Wexham Park Hospital, Slough, UK and University of Cyprus Medical School, Nicosia, Cyprus spaces throughout the supratentorial region and slumping of the brain stem. There was bilateral cerebellar herniation and dehydrated ventricular spaces with effacement of the cisterna magna (Fig 1b). MRI of the spine revealed an upper anterior cervical C1-C4 extradural fluid collection (Fig 1c). These findings were consistent with spontaneous intracranial hypotension resulting from a cerebrospinal fluid (CSF) leak.

Transthoracic echocardiography confirmed a tricuspid aortic valve with moderate central aortic regurgitation and mild aortic root dilatation. This raised the possibility of an underlying new diagnosis of HLA-B27-associated syndrome with aortic valve involvement, presenting without obvious rheumatic disease. The patient was treated with caffeine infusions and two thoracolumbar epidural blood patches over 3 months which provided transient relief. Over the next 2 months, there were features of behavioural variant frontotemporal dementia, with social disinhibition, unacceptable behaviour, loss of manners and decorum and impulsive actions. Dietary changes included an appetite for pork pies. He was now almost bedbound with daytime hypersomnolence (near narcolepsy) and there were episodes of dysphagia with choking.

The patient was referred for surgical intervention. Digital subtraction myelography demonstrated a left T8-T9 CSF-venous fistula. At operation, after removal of the left T8/9 lamina, a nerve root cyst was identified with a CSF venous fistula (VF) which was ligated with $11 \mathrm{~mm}$ aneurysm clips and repaired with a dural patch graft and fibrin glue.

Post-procedural MRI showed a reduction in the degree of cerebellar herniation, increased CSF space in the foramen magnum and better CSF volume at the cervicomedullary junction. There was a parallel resolution of the patient's symptoms within 7 days and dramatic improvement in his general behaviour over the following weeks.

A diagnosis of spontaneous intracranial hypotension (SIH) resulting from a CSF-VF was now made in the context of HLAB27-associated syndrome. Using the International Behavioural Variant Frontotemporal Dementia (bvFTD) Criteria Consortium, our patient fell in to the category of probable bvFTD based on the clinical syndrome (the presence of three of six behavioural and cognitive features: disinhibition, apathy/inertia, loss of empathy, perseverative/compulsive behaviours, hyperorality and a dysexecutive neuropsychological profile) plus demonstrable functional decline and frontotemporal imaging changes. ${ }^{1}$ 


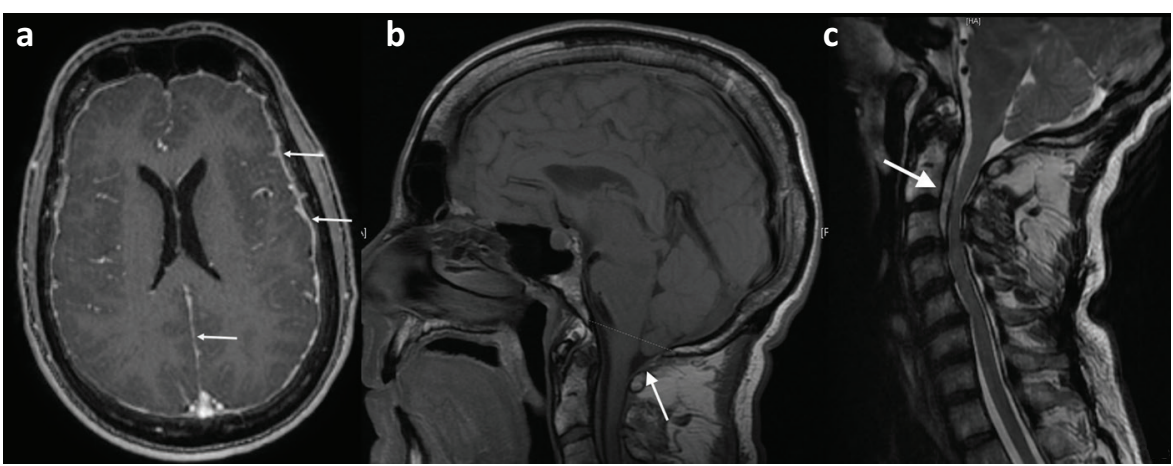

Fig 1. Magnetic resonance imaging of the brain and upper spine. a) Axial T1 weighted post contrast image demonstrating meningeal enhancement suggestive of pachymeningitis. b) Sagittal image showing features of spontaneous intracranial hypotension with tonsillar herniation. c) There was an anterior C1-C4 extradural fluid collection as a consequence of a cerebrospinal fluid leak; this was a false localising sign.

\section{Discussion}

SIH is characterised by a low-pressure orthostatic headache secondary to a reduction in the CSF pressure, without apparent provocation. Any breach in the dura mater causes a reduction in CSF volume leading to gravitational traction and downward displacement of the brainstem and cerebellum, hence the orthostatic nature of the headache.

The majority of patients have coinciding connective tissue disease (CTD) with dural weaknesses, spinal meningeal diverticula or perineural cysts (Tarlov cysts), all of which can predispose to spontaneous CSF leak. In our patient, the CSF leak likely resulted from unrecognised cumulative trauma to a pre-existing Tavlov cyst, a rare association of CTD. ${ }^{2}$

Dural defects predispose to the formation of CSF-VFs (with unregulated CSF loss) because of communications between the spinal subarachnoid space and dilated paraspinal veins that accompany SIH, a consequence of the Monro-Kellie rule. ${ }^{3,4}$

The characteristic MRI appearances of thickened pachymeninges and engorgement of dural venous sinuses, resulting from venous dilation in response to CSF volume reduction, are also in accordance with the Monro-Kellie hypothesis.

In our case, the epidural CSF collection seen in the upper cervical region was a false localising sign and distribution of CSF did not represent the site of leak, implying that pooling of CSF may be variably dependent on patient position and anatomy.

The loss of gag reflex and palatal sensation on presentation likely reflected brainstem compression of anatomically adjacent nuclei of cranial nerves X and XII, and normal pharyngeal reflexes returned on re-establishment of CSF volume.

The situation was further complicated by accelerated trajectory bvFTD. Conceivably this could have been resulted from prolonged intracranial hypotension and mechanical disruption of frontotemporal anatomy with structural, metabolic or neurodegenerative changes. This was apparently reversible on restoration of CSF volume. Our patient had shown features of bvFTD some 2 years prior to presentation, but there was a calamitous progression during the 6 months of radiologically proven SIH. It is possible that our patient had a period of subclinical SIH prior to presentation.

Most patients with SIH respond well to measures aimed at increasing CSF volume, including hydration with increased salt intake and the administration of caffeine, glucocorticoid medication or mineralocorticoid agents. Autologous epidural blood patches are safe and effective if there is failure in response to conservative management. Success rates are higher if the blood patch is administered directly at the extravasation site, particularly among patients for whom procedures at the lumbar level have been unsuccessful. Injection of fibrin glue as an alternative to blood patch has been used with some effect, and surgical intervention is saved as a last resort when a significant meningeal defect has been demonstrated.

\section{Conclusion}

SIH remains an important diagnostic consideration for persistent headache, and an index of suspicion should be exercised for concomitant CTD and dural weaknesses. SIH-associated acquired Chiari malformation is exceedingly rare, and we suspect our patient had a de novo diagnosis of CTD spectrum disorder and/ or HLA-B27-associated syndrome. The situation was further complicated by an unparalleled progression of bvFTD, potentially resulting from prolonged intracranial hypotension causing mechanical disruption of frontotemporal anatomy, which showed reversibility on restoration of CSF volume. To our knowledge, this is the first account SIH resulting from CSF venous fistulisation leading to a spectrum of neuropsychiatric features consistent with bvFTD.

\section{References}

1 Rascovsky K, Hodges JR, Knopman D et al. Sensitivity of revised diagnostic criteria for the behavioural variant of frontotemporal dementia. Brain 2011;134:2456-77.

2 Pabaney AH, Mirza FA, Syed NA et al. Spontaneous dural tear leading to intracranial hypotension and tonsillar herniation in Marfan syndrome: a case report. BMC Neurol 2010;10:54.

3 Kumar N, Diehn FE, Carr CM et al. Spinal CSF venous fistula: A treatable etiology for CSF leaks in craniospinal hypovolemia. Neurology 2016;86:2310-2.

4 Fishman RA, Dillon WP. Dural enhancement and cerebral displacement secondary to intracranial hypotension. Neurology 1993:43:609-11.

Address for correspondence: Prof Constantinos G Missouris, Departments of Cardiology and Internal Medicine, Wexham Park Hospital, Frimley Health NHS Foundation Trust, Wexham Street, Slough SL2 4HL, UK.

Email:dinos.missouris@nhs.net 